\title{
Privacy-Preserving Architecture for Forensic Image Recognition
}

\author{
Andreas Peter", Thomas Hartmann*, Sascha Müller", Stefan Katzenbeisser" \\ Security Engineering Group, TU Darmstadt and CASED \\ Mornewegstr. 32, 64293 Darmstadt, Germany \\ \# \{peter,mueller, katzenbeisser\}aseceng.informatik.tu-darmstadt.de \\ *ageartmann@web. de
}

\begin{abstract}
Forensic image recognition is an important tool in many areas of law enforcement where an agency wants to prosecute possessors of illegal images. The recognition of illegal images that might have undergone human imperceptible changes (e.g., a JPEG-recompression) is commonly done by computing a perceptual image hash function of a given image and then matching this hash with perceptual hash values in a database of previously collected illegal images. To prevent privacy violation, agencies should only learn about images that have been reliably detected as illegal and nothing else.

In this work, we argue that the prevalent presence of separate departments in such agencies can be used to enforce the need-toknow principle by separating duties among them. This enables us to construct the first practically efficient architecture to perform forensic image recognition in a privacy-preserving manner. By deriving unique cryptographic keys directly from the images, we can encrypt all sensitive data and ensure that only illegal images can be recovered by the law enforcement agency while all other information remains protected.
\end{abstract}

\section{INTRODUCTION}

The effective and efficient recognition of digitally stored data received much attention in the past [6], [10], [16], [17], [19], [20] or [22]. One of its main applications lies in the area of law enforcement where forensic data recognition helps in the prosecution of criminals. A common workflow of forensic investigations starts with a police force entering a private house and reading out all data stored on any data media found. Then, a separate department of the police station performs a matching of the found data with a collection of known illegal data. In practice, the matching is usually done by comparing cryptographic hash values of this data which reliably detects even a single bit change.

Regarding images, however, we are generally not interested in bit changes. For instance, a JPEG-recompressed image will have a completely different cryptographic hash value than the original image, while the two images are indistinguishable for the human eye. Consequently, we are rather interested in human imperceptible changes than single bit flips when dealing with digital images. A so-called perceptual hash function [11] is an efficient mean to detect these changes: perceptually similar images will produce similar hash values

WIFS'2012, December, 2-5, 2012, Tenerife, Spain.

978-1-4673-2287-4/12/\$31.00 (C)2012 IEEE. (e.g., with a small Hamming distance), while perceptually different images will produce hash values with a large Hamming distance. In the past, such hash functions have been successfully exploited in the area of law enforcement. For instance, Steinebach et al. [22] consider a scenario where a police force wants to prosecute criminals possessing images with child pornographic content. Concretely this is done by first computing perceptual hash values of all images found on any data media owned by the investigated person and then matching these hash values with a database consisting of perceptual hash values of previously collected illegal child pornographic images. Whenever the (Hamming) distance of an investigated hash value with a hash value in the database is below a certain image recognition threshold $t$, the investigated image will be detected as illegal.

This setting can be formulated in more general terms: two independent departments of a law enforcement agency work together in order to prosecute possessors of images with illegal content. The first department starts the investigation by using a device, which we call the preprocessing device. This device has direct/physical access to the data media storing (private) images and performs some preprocessing on the original data (in [22], this device simply copies all data to another data media, like a hard disk). Thereafter, a matching device of the second department, with access to the preprocessed data from the preprocessing device (and not to the original, unprocessed data), matches each preprocessed data item with a previously generated database containing illegal images in order to recognize illegal content.

In such settings, all data (including very sensitive data) is available to both departments in the clear, which poses a huge violation to the investigated person's privacy. Assume, for instance, that the suspect possesses no illegal data whatsoever, still both departments will have access to all his data (including his most sensitive data) although he is completely innocent. From a privacy perspective, this circumstance is utterly unacceptable.

In this work, we present a simple and efficient solution to prosecute a suspect in the above setting, while preserving the suspect's privacy on sensitive data which is unrelated to the actual criminal case. We argue that by separating duties among the two existing departments (the preprocessing and the matching device) of the law enforcement agency, 
we can enforce the need-to-know principle and thus reduce privacy risks to a minimum. Since all our techniques can be implemented in copying hardware, it makes sense to treat the preprocessing device as a trusted party with physical access to the suspect's data, which sanitizes the original data from all sensitive information. Note that in practice there is always a party (preprocessing device) with direct access to the suspect's data, so we are forced to trust this party. The preprocessed (sanitized) data can then securely been given to the untrusted matching device which only learns about the reliably detected illegal images owned by the suspect and nothing else. To prove the practical efficiency of our solution, we give a proof-ofconcept implementation and evaluate its performance.

Related Work. Concerning the construction and use of perceptual hash functions in different scenarios, we refer to [20] for an overview on techniques. Most interesting for our purposes is the work by Steinebach et al. [22] (and the references therein) that successfully uses perceptual hash functions in the area of law enforcement.

We note that our work heavily relies on a cryptographic primitive, called Fuzzy Extractor [9]. This primitive reliably extracts a uniformly random key from a biometric input together with certain helper data, which later on assists in reconstructing the same key without knowing the original input. Fuzzy extractors have been extensively used in the area of biometric template protection [12] (and [21]), while paying much attention to iris-based [7] (and [8]), fingerprintbased [14] (and [15]), and face-based [23] templates.

Outline. We recall the basic building blocks used for our construction in Section II and present our protocol for the privacypreserving image recognition in Section III. Implementation details are dealt with in Section IV, where we will also discuss concrete parameter choices for our construction. We conclude with potential further application scenarios in Section V.

\section{PRELIMINARIES}

We make use of the following four building blocks: cryptographic hash functions, perceptual hash functions, fuzzy extractors, and symmetric-key encryption. As usual [18, Ch. 1.9], we call a hash function $\mathrm{cH}_{r}:\{0,1\}^{*} \longrightarrow\{0,1\}^{r}(r \in \mathbb{N}$ fixed) cryptographic if it is pre-image resistant, collision resistant, and unpredictable. We treat cryptographic hash functions $\mathrm{CH}_{r}$ as random oracles [5] which ensures that the outputs are uniformly distributed in $\{0,1\}^{r}$.

Perceptual Hash Functions. We define a perceptual (image $)^{1}$ hash function as a deterministic compression function $\mathrm{pH}_{n}^{t}:\{0,1\}^{*} \longrightarrow\{0,1\}^{n}$ such that perceptually similar images yield outputs with a small Hamming distance ${ }^{2}$, say $\leq t$. So two given images $\mathrm{img}_{1}$ and $\mathrm{img}_{2}$ are perceptually similar if $\Delta_{H}\left(\mathrm{pH}_{n}^{t}\left(\mathrm{img}_{1}\right), \mathrm{pH}_{n}^{t}\left(\mathrm{img}_{2}\right)\right) \leq t$ where $\Delta_{H}(\cdot, \cdot)$

\footnotetext{
${ }^{1}$ Note that perceptual hash functions can be defined for different kinds of media objects and not only for images. In this work, however, we are only interested in perceptual hash functions with respect to images.

${ }^{2}$ We focus on the Hamming distance here, although other distance functions can be used.
}

denotes the Hamming distance function. There are numerous instantiations of such perceptual hash functions (see [25] for an overview) which are based on different techniques. For more details on perceptual hash functions, we refer to [20].

We stress that perceptual hash functions are error-prone depending on the size of the image recognition threshold $t$ : perceptual hashes of perceptually similar images may have a Hamming distance greater than $t$ and will thus be detected as different (false rejection), while the hashes of perceptually different images may have a Hamming distance smaller or equal to $t$ and will thus be detected as similar (false acceptance). For greater choices of $t$, we will have a lower false rejection rate (FRR) but a higher false acceptance rate (FAR), and vice versa. When applying perceptual hash functions in areas where the detection of illegal images has serious consequence (e.g., in the area of law enforcement), we need to make sure that detected images are crosschecked by the human eye. Furthermore, it is not necessary to detect all illegal images but "sufficiently many" for criminal conviction.

Fuzzy Extractors. As explained in the previous paragraph, a perceptual hash function will not produce the same hash value for preceptually similar images but will produce values having a small (pairwise) Hamming distance of $\leq t$. A Fuzzy Extractor (FE) [9] offers a way to circumvent this issue: for a given perceptual hash of an image, the FE produces a uniformly random string $K$ together with a certain public helper data $h$ in an enrolment phase. Later on, this helper data can be used in a reconstruction phase to produce the same string $K$ when given the perceptual hash of an image that is perceptually similar to the original image. It is important to note that the string $K$ remains uniformly random even when given the helper data $h$. Concretely, an FE can be instantiated as follows:

In a setup phase, an error-correcting binary ${ }^{3}$ linear $[\mu, k, d]$ code $\mathcal{C}$ of bit length $\mu$, cardinality $2^{k}$, and minimum distance $d$ is chosen. Due to the choice of parameters, the code can correct up to $\left\lfloor\frac{d-1}{2}\right\rfloor$ errors. There are many known ways to construct such codes for given parameters [9]. When applying the FE to perceptual hash values in $\{0,1\}^{n}$, we need to make sure that the bit length $\mu$ of the code $\mathcal{C}$ coincides with the bit length $n$ of the output of the hash function, and that the number $\left\lfloor\frac{d-1}{2}\right\rfloor$ of correctable errors is greater or equal to the image recognition threshold $t$ of the perceptual hash function.

In the enrolment phase, denoted by FE.Gen, given a perceptual hash value $\mathrm{pH}_{n}^{t}$ (img) of an image img, we choose a codeword $\gamma \in \mathcal{C}$ uniformly at random and compute the helper data $h$ as $h=\gamma \oplus \mathrm{pH}_{n}^{t}$ (img). In order to get a uniformly random string $K$, we apply a cryptographic hash function $\mathrm{cH}_{r}:\{0,1\}^{*} \longrightarrow\{0,1\}^{r}$ to $\gamma$, i.e., $K=\mathrm{cH}_{r}(\gamma)$.

Later, during the reconstruction phase (denoted by FE.Rep), for any given perceptual hash value $\mathrm{pH}_{n}^{t}\left(\mathrm{img}^{\prime}\right)$ of Hamming distance $\leq t$ with $\mathrm{pH}_{n}^{t}$ (img) (i.e., $\mathrm{img}^{\prime}$ and img are perceptually similar) and given helper data $h$ (corresponding to

\footnotetext{
${ }^{3}$ We restrict our attention to binary codes (i.e., codes over the binary Galois field $\mathbb{F}_{2}$ ). However, the same discussion can also be done for non-binary codes.
} 
$\left.\mathrm{pH}_{n}^{t}(\mathrm{img})\right)$, we first compute $W:=\mathrm{pH}_{n}^{t}\left(\mathrm{img}^{\prime}\right) \oplus h$, and then use the decoding algorithm of the error correcting code $\mathcal{C}$ on $W$, which outputs the same codeword $\gamma$ that we randomly picked in the enrolment phase. Then, applying $\mathrm{cH}_{r}$ to this codeword, we reconstruct the string $K=\mathrm{cH}_{r}(\gamma)$.

\section{Privacy-Preserving Image ReCognition}

The Setting. We recall the abstract scenario set forth in Section I:

- Given a data media $\mathcal{M}$ storing all kinds of images, a matching of these images with a database $\mathcal{D}$ consisting of illegal images is to be performed. As described in Section I, such matchings are very important in the area of law enforcement.

- A trusted preprocessing device with physical access to $\mathcal{M}$ performs some preprocessing on the images stored on $\mathcal{M}$.

- A separate untrusted matching device receives the preprocessed data from the preprocessing device with no access to the original data media $\mathcal{M}$ and matches this data with the database $\mathcal{D}$ in order to detect illegal images in the preprocessed data.

- The goal is to make the matching process privacypreserving: The matching device should only learn those images that are reliably detected to be illegal and no other information.

High-Level Description of our Construction. Recall that we want to enforce the need-to-know principle by separating duties among the preprocessing and the matching device. Consequently, each device should only learn the information it really needs to know. Since the preprocessing device is trusted, we require it to modify the original data media $\mathcal{M}$ in such a way that the resulting preprocessed data poses no privacy threat when giving it to the untrusted matching device. Before any processing is done at all, there is an initial setup that sets up the following building blocks:

- a perceptual hash function $\mathrm{pH}_{n}^{t}:\{0,1\}^{*} \longrightarrow\{0,1\}^{n}$ of bit length $n$ and image recognition threshold $t$,

- an error-correcting binary linear $[\mu, k, d]$-code $\mathcal{C}$ such that $\mu=n$ and $\left\lfloor\frac{d-1}{2}\right\rfloor \geq t$ (used in the FE),

- a cryptographic hash function $\mathrm{cH}_{r}:\{0,1\}^{*} \longrightarrow\{0,1\}^{r}$ of bit length $r$ such that $r \leq k$ (used in the FE) ${ }^{4}$, and

- a symmetric-key encryption scheme with encryption function Enc and decryption function Dec that can handle variable sized plaintext messages, along with a key derivation function KDF for this particular encryption scheme. We denote encryption of a message $m$ under a symmetric key $K$ by $\operatorname{Enc}_{K}(m)$ and decryption of a ciphertext $c$ under $K$ by $\operatorname{Dec}_{K}(c)$.

\footnotetext{
${ }^{4}$ We require that $r \leq k$ since we want the output of the FE to be uniformly random. Recall that the FE will apply the cryptographic hash function to a uniformly random codeword. This codeword is drawn from an $n$-bit code with $2^{k}$ different codewords, meaning that a randomly chosen $n$-bit codeword will have $k$ bits of entropy. So requiring $r \leq k$ ensures an entropy of $r$ bits in the output of the hash function.
}

We give concrete instantiations of the above building blocks in Section IV. Now, on a high-level, we let the preprocessing device perform the following steps for each image img on the media $\mathcal{M}$ :

1) Compute the perceptual hash $\mathrm{pH}_{n}^{t}(\mathrm{img})$ and give it as input to an algorithm that we call the sanitizer.

2) On input the perceptual hash $\mathrm{pH}_{n}^{t}(\mathrm{img})$, the sanitizer outputs a cryptographic key $K$ (for the symmetrickey encryption scheme), helper data $h$, and a sanitized version of the perceptual hash that we denote by $\mathrm{SpH}_{\mathrm{r}}(\mathrm{img})$. This sanitized version is completely free of all private information stored in img (in fact, we will see that it is indistinguishable from a uniformly random string).

3) The cryptographic key $K$ is then used in the symmetrickey encryption function Enc in order to encrypt the image img itself and some potential meta-data meta which may include the filename, path information, or other file information such as timestamps, attributes, etc.

4) The encrypted image (and meta-data) $\mathrm{Enc}_{K}$ (img, meta), the helper data $h$, and the sanitized perceptual hash $\mathrm{SpH}_{\mathrm{r}}(\mathrm{img})$ is the preprocessed data which can now safely be given to the untrusted matching device (e.g., on an external hard disk).

These steps are depicted in Figure 1 on the left hand side of the dashed "separation of duty"-line (preprocessing device).

Once the preprocessing device processed all images on the data media $\mathcal{M}$ in the above described steps, the untrusted matching device performs the following steps for each preprocessed image data $\left(\mathrm{Enc}_{K}\left(\mathrm{img}\right.\right.$, meta) $\left., h, \mathrm{SpH}_{\mathrm{r}}(\mathrm{img})\right)$ :

1) For each illegal image in the public database $\mathcal{D}$, an extractor uses the helper data $h$ in order to compute a hash-extract hext that is then matched (in a matching algorithm) with the sanitized perceptual hash $\mathrm{SpH}_{\mathrm{r}}(\mathrm{img})$ from the preprocessed image data in a privacy-preserving manner.

2) The matching algorithm takes the sanitized perceptual hash $\mathrm{SpH}_{\mathrm{r}}(\mathrm{img})$ and the hash-extract hext as input and computes a cryptographic key $K^{\prime}$. If the matching was successful, the key $K^{\prime}$ coincides with the symmetric key $K$ under which the image img and the meta-data meta was encrypted, and so this data can be recovered simply by decrypting. If the matching failed, the whole process starts all over again, taking the next illegal image of the database $\mathcal{D}$ as input.

For a pictorial overview on these steps, see the right hand side of the dashed "separation of duty"-line (matching device) in Figure 1.

We note that encryption (in the preprocessing) and decryption (in the matching) are actually not needed for the matching to work (the sanitized perceptual hashes and the helper data are sufficient). Still, we include these two procedures in order for the matching device to crosscheck the suspected illegal images by the human eye (recall that perceptual hash functions are error-prone, see Section II). 


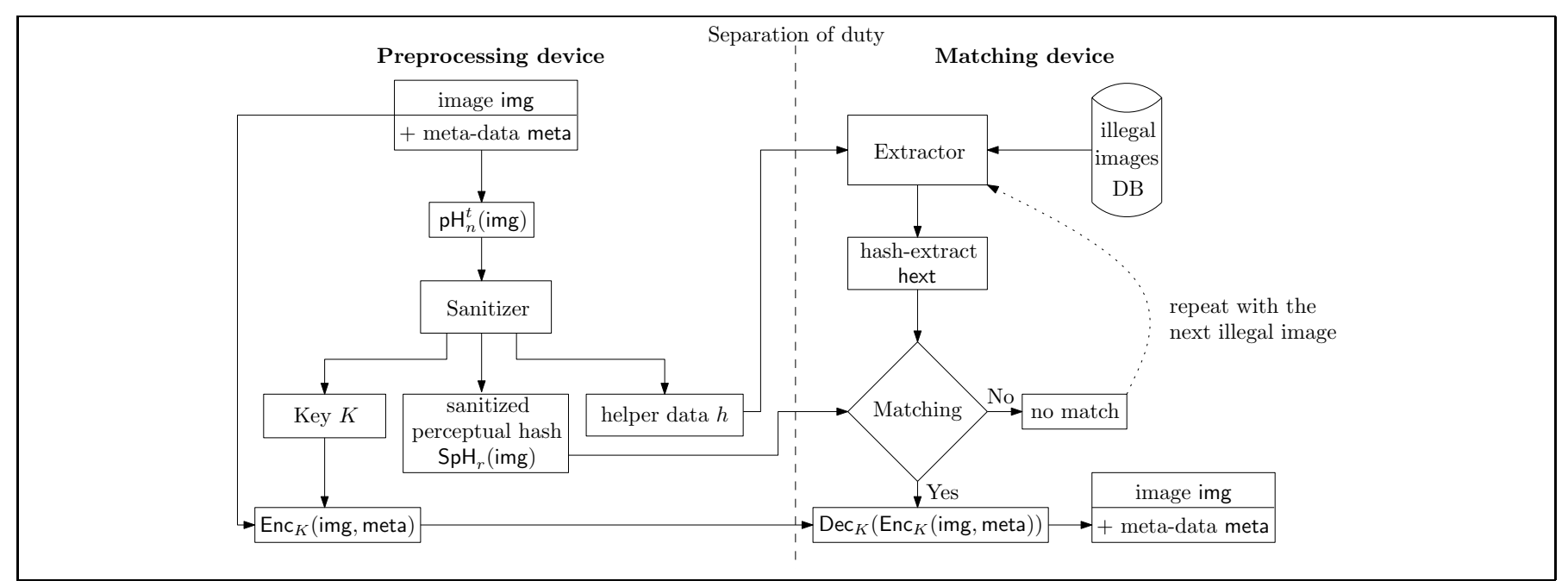

Fig. 1. Pictorial high-level description of our construction.

In the following, we give the technical details of the three main algorithms used in the above steps: the sanitizer at the preprocessing device, as well as the extractor and the matching at the matching device.

The Sanitizer. The sanitizer algorithm takes a perceptual hash $\mathrm{pH}_{n}^{t}$ (img) of an image img as input and outputs a cryptographic key $K$ (for the symmetric-key encryption scheme), helper data $h$, and a sanitized version $\mathrm{SpH}_{\mathrm{r}}(\mathrm{img})$ of the perceptual hash $\mathrm{pH}_{n}^{t}$ (img). The output is computed as described in Algorithm 1.

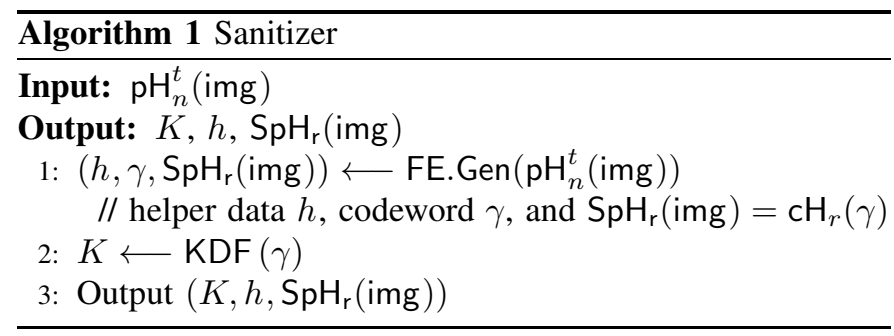

The Extractor. The extractor algorithm takes helper data $h$ (corresponding to an image img) and the perceptual hash $\mathrm{pH}_{n}^{t}\left(\mathrm{img}_{\text {ill }}\right)$ of an illegal image $\mathrm{img}_{\text {ill }}$ of the public database $\mathcal{D}$ as input and outputs the hash-extract hext. The hashextract consists of two components. The first component is a codeword that essentially is a "correction" of the perceptual hash of $\operatorname{img}_{i l l}$ by using $h$ that can be used to recover the decryption key if img $_{i l l}$ is perceptually similar to img. The second component is the cryptographic hash function $\mathrm{cH}_{r}$ applied to this "correction", which transforms it into a format so that it can be easily matched with the suspected sanitized hash $\mathrm{pH}_{n}^{t}$ (img) of img later on in the matching algorithm. The individual steps of this procedure are described in Algorithm 2.

The Matching. The matching algorithm takes a sanitized perceptual hash $\mathrm{SpH}_{\mathrm{r}}(\mathrm{img})$ and a hash-extract hext as input

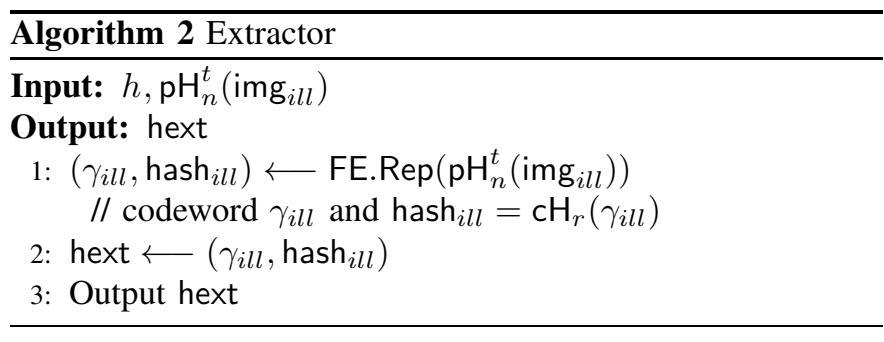

and outputs either a cryptographic key $K^{\prime}$ or the symbol $\perp$. The key $K^{\prime}$ will coincide with the decryption $K$ if and only if the matching was successful. This is done by performing the steps of Algorithm 3.

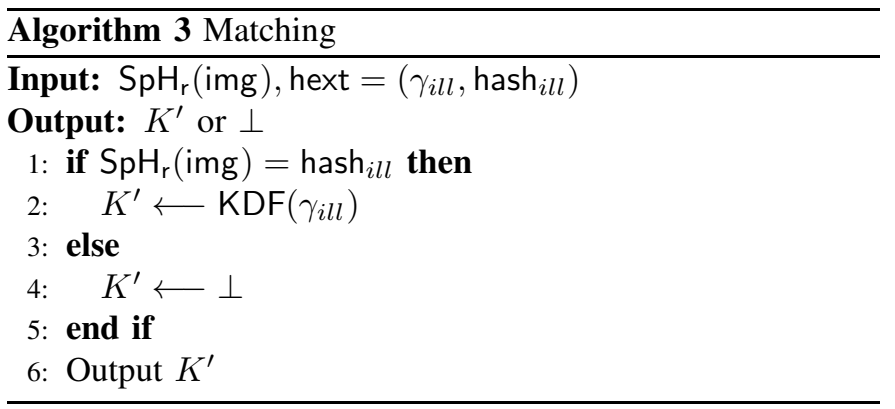

Correctness and Security. Assume that the preprocessing device finished its computation for a given image img (with meta-data meta) and sends the resulting triplet (Enc $K$ (img, meta), $h, \mathrm{SpH}_{\mathrm{r}}$ (img)) to the matching device. For each image $\mathrm{img}_{\text {ill }}$ in the public database $\mathcal{D}$ of illegal images, we have the following distinction of cases:

1) The image img is perceptually similar to the illegal image $\mathrm{img}_{\text {ill }}$. This means that the Hamming distance between the perceptual hashes of the two images img and $\mathrm{img}_{i l l}$ is less or equal to the image recognition threshold $t$. By the properties of the FE (cf. Section II), the algorithm FE. $\operatorname{Rep}\left(\mathrm{pH}_{n}^{t}\left(\mathrm{img}_{i l l}\right)\right)$ will produce the 
same codeword $\gamma$ as the algorithm FE.Gen $\left(\mathrm{pH}_{n}^{t}(\mathrm{img})\right)$ since the used error-correcting code $\mathcal{C}$ corrects all $t$ errors and will decode to the same codeword $\gamma$. This implies that hash $i l l=\mathrm{SpH}_{\mathrm{r}}(\mathrm{img})$ and so the output hext of the extractor coincides with $\left(\gamma, \mathrm{SpH}_{\mathrm{r}}(\mathrm{img})\right)$. This in turn means that the IF-statement in the matching algorithm evaluates to TRUE and thus produces the key $K^{\prime}=\operatorname{KDF}(\gamma)$. Obviously, we have $K^{\prime}=K$ and so the matching device can successfully decrypt $\mathrm{Enc}_{K}$ (img, meta).

2) The image img is perceptually different from the illegal image $\mathrm{img}_{\text {ill }}$. This means that the Hamming distance between the perceptual hashes of the two images img and $\mathrm{img}_{\text {ill }}$ is greater than the image recognition threshold $t$. Then, the decoding of $\mathrm{pH}_{n}^{t}\left(\mathrm{img}_{i l l}\right) \oplus h$ will yield a codeword $\gamma_{i l l}$ that is different from $\gamma$ (see also [9]). Therefore, the hash value hash $i l l=\mathrm{cH}_{r}\left(\gamma_{i l l}\right)$ will differ from $\mathrm{SpH}_{\mathrm{r}}(\mathrm{img})$ and the IF-statement in the matching algorithm will evaluate to FALSE and thus output the symbol $\perp$. In this case, decryption of $\mathrm{Enc}_{K}$ (img, meta) is impossible. This is ensured by the security properties of the FE (cf. [9]): Without knowing a perceptual hash that is within a Hamming distance of $\leq t$ to $\mathrm{pH}_{n}^{t}(\mathrm{img})$, the hash $\mathrm{pH}_{n}^{t}(\mathrm{img}$ ) (and its corresponding image img) stays information-theoretically hidden.

We stress that the only information the untrusted matching device sees is the triplet $\left(\mathrm{Enc}_{K}(\mathrm{img}, \mathrm{meta}), h, \mathrm{SpH}_{\mathrm{r}}(\mathrm{img})\right)$. The first component is a secure encryption and is hence indistinguishable from a uniformly random value. The second two components $\left(h, \mathrm{SpH}_{\mathrm{r}}(\mathrm{img})\right)$ are indistinguishable from random as well by the properties of the FE (cf. Section II). As the preprocessing device is trusted, this shows that our protocol indeed is privacy-preserving in the sense that if an image is perceptually different from all illegal images in the database $\mathcal{D}$, the matching device learns no information whatsoever, except for a couple of uniformly random strings (independent of the original image).

\section{IMPLEMENTATION}

Instantiating the Protocol. We instantiate the building blocks needed in our protocol, as described in Section III, as follows:

- As perceptual hash function $\mathrm{pH}_{n}^{t}:\{0,1\}^{*} \longrightarrow\{0,1\}^{n}$ of bit length $n$ and image recognition threshold $t$, we use the block mean value based hash function by Yang et al. [24] with parameters $n=960$ and $t=12$ for efficiency and implementation reasons (more precisely, we use the second method proposed in [24]). Note that this particular hash function has been successfully used to recognize images in the forensic context [22]. Therein, they evaluated that an image recognition threshold of $t=$ 12 suffices to reliably identify illegal images with an FRR of $\approx 4.6 \%$ and an FAR of $\approx 0 \%$ when working with a sample set of certain "cheerleading event" images (see "performance analysis" below for more details). In our actual implementation, we rely on the open source library
pHash [4] that provides an efficient implementation of this block mean value based hash function. The computed hashes have the fixed bit length of $n=960$ bits. We note that in order to handle compressed image formats, the pHash library uses the open source CImg Library [1] for image processing.

- We instantiate the FE with the recommended parameter setting of [9] and rely on the recommended (freely available) implementation by Morelos-Zaragoza [2] of an error-correcting binary linear $[\mu, k, d]$-code $\mathcal{C}$ such that $\mu=n$ and $\left\lfloor\frac{d-1}{2}\right\rfloor \geq t$. More precisely, [2] implements a $[\mu=960, k=840, d=25]-\mathrm{BCH}$ code along with an efficient decoding algorithm.

- As cryptographic hash function $\mathrm{cH}_{r}:\{0,1\}^{*} \longrightarrow\{0,1\}^{r}$ of bit length $r$ such that $r \leq k$, we use SHA512 from the Mhash library [3], i.e., $r=512 \leq k=840$. Together with our choice of the error-correcting code, we get a secure instantiation of the FE (cf. [9]).

- As a symmetric-key encryption scheme that can handle variable sized plaintext messages, we use a standard binary additive stream cipher [18] and use the PKCS\#1 [13, Appendix B.2.1] mask generation function (i.e., a keystream generator for the used stream cipher) $\mathrm{MGF}_{\ell}:\{0,1\}^{*} \longrightarrow\{0,1\}^{\ell}$ as the key derivation function KDF, where $\ell$ is a second input and is chosen as the bit length of the message to be encrypted.

Performance Analysis. With the above described instantiation of the protocol, the performance depends on the number $L$ of images processed by the preprocessing device and the number $I$ of illegal perceptual hash values stored in the public database $\mathcal{D}$. We analyzed the performance of the preprocessing device and the matching device separately, and ran all tests on an MS Windows XP Professional system with an Intel Core 2 Duo Processor E8400 running at $3 \mathrm{GHz}$. As sample images, we took 4,400 images of "cheerleading events" (which have similar characteristics as pornographic images) saved in JPEG format with file sizes ranging from $9 \mathrm{~KB}$ to $44 \mathrm{~KB}$. We computed the perceptual hashes of the first $I=2,200$ of these images and stored the hashes in the database $\mathcal{D}$. We then constructed a "fake criminal" by storing all $L=4,400$ images, JPEG-recompressed with a scaling factor that ensured the larger edge to be 300 pixels long, meaning an average size reduction of $25 \%$ compared to the original images and JPEG quality factor of 20 in a dedicated folder on the system. This is the same set of images and modifications used in the evaluation of [22]. This allowed us to verify their result that shows an FRR of $\approx 10 \%$ and an FAR of $\approx 0 \%$ with an image recognition threshold of $t=8$, while we have an FRR of $\approx 4 \%$ and an FAR of $\approx 0 \%$ with $t=12$.

For the preprocessing device, computing the perceptual hash of a given image took the most amount of time: $107.98 \mathrm{~ms}$ (on average). The whole process of computing the preprocessed data $\left(\mathrm{Enc}_{K}\left(\mathrm{img}\right.\right.$, meta)$\left., h, \mathrm{SpH}_{\mathrm{r}}(\mathrm{img})\right)$ including the storage of this data in a separate folder took $111.93 \mathrm{~ms}$ per image (on average). In total, the preprocessing device needed 8.2 minutes 
for all images.

For the matching device, the running time will depend on whether a given image is perceptually similar to an image in the database $\mathcal{D}$ and where the corresponding illegal image is positioned in the database. Therefore, we decided to give an upper bound on the running time of the matching device by considering only images that cannot be found in the database and so we always have to do a matching with all illegal images in the database per given suspected image. Recall that the only difference between running the protocol with a legal image and running it with an illegal image, is that we compute the key derivation function KDF in the matching algorithm. To capture the running time of this as well, we included a "fake" call of KDF with a random codeword. The timing results of this analysis are summarized in Table I.

\begin{tabular}{|l|l|l|l|}
\hline $\begin{array}{l}\text { \# of suspected } \\
\text { images }(L)\end{array}$ & $\begin{array}{l}\text { \# of images } \\
\text { in } \mathcal{D}(I)\end{array}$ & $\begin{array}{l}\text { total time, } \\
\text { in min }\end{array}$ & $\begin{array}{l}\text { avg. time/susp. } \\
\text { img, in ms }\end{array}$ \\
\hline 2,200 & 2,200 & 15.83 & 431.69 \\
\hline 2,200 & 6,600 & 47.34 & $1,291.08$ \\
\hline 4,400 & 2,200 & 31.59 & 430.79 \\
\hline 4,400 & 6,600 & 94.78 & $1,292.41$ \\
\hline 4,400 & 13,200 & 189.34 & $2,581.91$ \\
\hline
\end{tabular}

TABLE I

RUNNING TIMES OF THE MATCHING DEVICE FOR DIFFERENT AMOUNTS OF LEGAL AND ILLEGAL IMAGES. THE AMOUNT OF 2,200 LEGAL (L) AND ILLEGAL (I) IMAGES HAS BEEN ADAPTED TO THE RESPECTIVE AMOUNT BY DUPLICATING OR REMOVING RANDOMLY CHOSEN IMAGES THEREOF.

We note that the average time for a matching of a given suspected image and a given perceptual hash in the database $\mathcal{D}$ is $0.2 m s$.

\section{CONCLUSION}

In scenarios, where two departments of a law enforcement agency work together to prosecute possessors of illegal images (e.g., with child pornographic content as in the setting of [22]), we argued that very sensitive data (including data that is not even related to the actual criminal case) will be given to the agency in the clear, thus posing a huge privacy violation. To circumvent this, we introduced a protocol that exploits the existence of two separate departments in order to perform the image recognition in a privacy-preserving manner. We have seen that the protocol runs efficiently on our chosen sample images which makes it employable in real-world scenarios.

The basic idea of our protocol for privacy-preserving image recognition is not limited to the actual use of images. In fact, there are a lot more examples where law enforcement agencies need access to private data of suspected persons, but they are juridically not allowed to see data which is not relevant to the criminal case. One example for this lies in the area of illegal bank transactions. Of course, a different type of "robust" hash that is tailored to bank transactions would be required in order to challenge this problem. We leave this as interesting future work.

\section{ACKNOWLEDGMENT}

This work was funded by Hessen ModellProjekte (HAproject 243/10-19) within LOEWE - Landes-Offensive zur Entwicklung Wissenschaftlich-ökonomischer Exzellenz.

\section{REFERENCES}

[1] The cimg library: A c++ template image processing toolkit. http://cimg. sourceforge.net/.

[2] Implementation of a [960, 840, 25]-bch code. http://www.eccpage.com/ bch3.c/.

[3] Mhash: An open source hash library. http://mhash.sourceforge.net/.

[4] Phash: the open source perceptual hash library. http://www.phash.org/.

[5] Mihir Bellare and Phillip Rogaway. Random oracles are practical: A paradigm for designing efficient protocols. In $C C S$ '93, pages 62-73. ACM, 1993.

[6] Sushil K. Bhattacharjee and Martin Kutter. Compression tolerant image authentication. In ICIP (1), pages 435-439, 1998.

[7] George I. Davida, Yair Frankel, and Brian J. Matt. On enabling secure applications through off-line biometric identification. In IEEE Symposium on Security and Privacy, pages 148-157. IEEE, 1998.

[8] George I. Davida, Yair Frankel, Brian J. Matt, and Ren Peralta. On the relation of error correction and cryptography to an off line biometric based identification scheme. In Proc. of WCC99, pages 129-138, 1999.

[9] Yevgeniy Dodis, Rafail Ostrovsky, Leonid Reyzin, and Adam Smith. Fuzzy extractors: How to generate strong keys from biometrics and other noisy data. SIAM J. Comput., 38(1):97-139, 2008.

[10] Jiri Fridrich. Robust bit extraction from images. In ICMCS, Vol. 2, pages 536-540, 1999

[11] Jiri Fridrich. Robust hash functions for digital watermarking. In ITCC'00, pages 178-183. IEEE, 2000.

[12] Anil K. Jain, Karthik Nandakumar, and Abhishek Nagar. Biometric template security. EURASIP J. Adv. Sig. Proc., 2008, 2008.

[13] RSA Laboratories. PKCS \# 1 v2.1: RSA cryptography standard, 2002.

[14] Qiming Li and Ee-Chien Chang. Robust, short and sensitive authentication tags using secure sketch. In $M M \& S e c ' 06$, pages 56-61, 2006.

[15] Qiming Li, Muchuan Guo, and Ee-Chien Chang. Fuzzy extractors for asymmetric biometric representations. In IEEE Workshop on Biometrics (in association with CVPR), pages 1-6. IEEE, 2008.

[16] Ching-Yung Lin and Shih-Fu Chang. A robust image authentication method distinguishing jpeg compression from malicious manipulation. IEEE Trans. Circuits Syst. Video Techn., 11(2):153-168, 2001.

[17] Albert Meixner and Andreas Uhl. Robustness and security of a waveletbased cbir hashing algorithm. In $M M \& S e c$, pages 140-145. ACM, 2006.

[18] Alfred Menezes, Paul C. van Oorschot, and Scott A. Vanstone. Handbook of Applied Cryptography. CRC Press, 1996.

[19] Mehmet Kivanç Mihçak and Ramarathnam Venkatesan. New iterative geometric methods for robust perceptual image hashing. In Digital Rights Management Workshop, volume 2320 of LNCS, pages 13-21. Springer, 2001.

[20] Vishal Monga. Perceptually Based Methods for Robust Image Hashing. $\mathrm{PhD}$ thesis, The University of Texas at Austin, 2005.

[21] Christian Rathgeb and Andreas Uhl. A survey on biometric cryptosystems and cancelable biometrics. EURASIP J. Information Security, 2011:3, 2011.

[22] Martin Steinebach, Huajian Liu, and York Yannikos. Forbild: efficient robust image hashing. In Media Watermarking, Security, and Forensics, volume 8303 of Proc. SPIE. SPIE, 2012.

[23] Yagiz Sutcu, Qiming Li, and Nasir D. Memon. Protecting biometric templates with sketch: Theory and practice. IEEE Transactions on Information Forensics and Security, 2(3-2):503-512, 2007.

[24] Bian Yang, Fan Gu, and Xiamu Niu. Block mean value based image perceptual hashing. In IIH-MSP, pages 167-172. IEEE, 2006.

[25] Christoph Zauner. Implementation and benchmarking of perceptual image hash functions. Master's thesis, Upper Austria University of Applied Sciences, 2010. http://www.phash.org/docs/pubs/thesis_zauner.pdf. 